\title{
Adaptation of pineal expressed teleost exo-rod opsin to non-image forming photoreception through enhanced Meta II decay
}

\author{
Emma E. Tarttelin • Maikel P. Fransen • Patricia C. Edwards • \\ Mark W. Hankins • Gebhard F. X. Schertler • Reiner Vogel • Robert J. Lucas • \\ James Bellingham
}

Received: 24 November 2010/Revised: 1 February 2011/Accepted: 1 March 2011/Published online: 17 March 2011

(C) The Author(s) 2011. This article is published with open access at Springerlink.com

\begin{abstract}
Photoreception by vertebrates enables both image-forming vision and non-image-forming responses such as circadian photoentrainment. Over the recent years, distinct non-rod non-cone photopigments have been found to support circadian photoreception in diverse species. By allowing specialization to this sensory task a selective advantage is implied, but the nature of that specialization remains elusive. We have used the presence of distinct rod opsin genes specialized to either image-forming (retinal rod opsin) or non-image-forming (pineal exo-rod opsin) photoreception in ray-finned fish (Actinopterygii) to gain a unique insight into this problem. A comparison of biochemical features for these paralogous opsins in two model
\end{abstract}

E. E. Tarttelin and M. P. Fransen contributed equally to this work and should be considered joint first authors.

Electronic supplementary material The online version of this article (doi:10.1007/s00018-011-0665-y) contains supplementary material, which is available to authorized users.

E. E. Tarttelin · R. J. Lucas $(\bowtie)$

Faculty of Life Sciences, The University of Manchester, AV Hill Building, Oxford Road, Manchester M13 9PT, UK e-mail: robert.lucas@manchester.ac.uk

M. P. Fransen · P. C. Edwards · G. F. X. Schertler Structural Studies, MRC Laboratory of Molecular Biology, Hills Road, Cambridge CB2 0QH, UK

M. W. Hankins

Nuffield Laboratory of Ophthalmology, University of Oxford, John Radcliffe Hospital, Headley Way, Oxford OX3 9DU, UK

R. Vogel $(\bowtie)$

Biophysics Section, Institute of Molecular Medicine and Cell Research, University of Freiburg,

Hermann-Herder-Strasse 9, 79104 Freiburg, Germany

e-mail: reiner.vogel@biophysik.uni-freiburg.de teleosts, Fugu pufferfish (Takifugu rubripes) and zebrafish (Danio rerio), reveals striking differences. While spectral sensitivity is largely unaltered by specialization to the pineal environment, in other aspects exo-rod opsins exhibit a behavior that is quite distinct from the cardinal features of the rod opsin family. While they display a similar thermal stability, they show a greater than tenfold reduction in the lifetime of the signaling active Meta II photoproduct. We show that these features reflect structural changes in retinal association domains of helices 3 and 5 but, interestingly, not at either of the two residues known to define these characteristics in cone opsins. Our findings suggest that the requirements of non-image-forming photoreception have lead exo-rod opsin to adopt a characteristic that seemingly favors efficient bleach recovery but not at the expense of absolute sensitivity.

Keywords Rod opsin · Exo-rod · Rhodopsin . GPCR $\cdot$ Teleost $\cdot$ Circadian

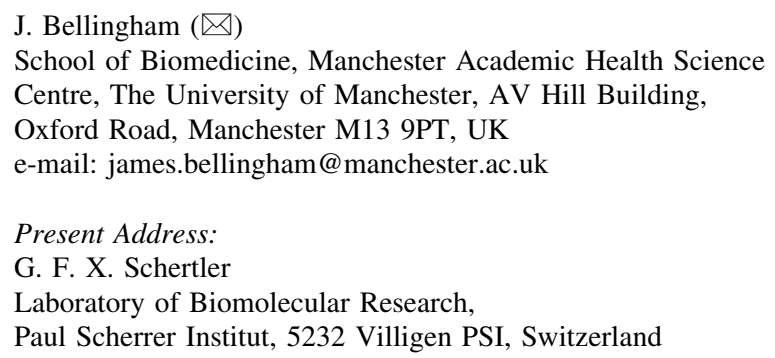




\section{Introduction}

The first step in vertebrate vision is the absorption of light by rod and cone photoreceptors. This is achieved by photopigments comprising either a rod or cone opsin protein binding a vitamin A-derived retinaldehyde-based chromophore. Opsins are members of the G-protein coupled receptor superfamily of proteins. In the dark, they bind the $\mathrm{A}_{1}$ chromophore 11-cis retinaldehyde in their ligandbinding pocket covalently via a protonated Schiff base. This chromophore acts as an inverse agonist suppressing G-protein activation, but can be isomerized to the agonist (all-trans conformation) by light [1]. As a result, opsins show light-dependent interaction with their G-protein signaling cascade. The opsin expressed in rods (rod opsin) was the first G-protein coupled receptor to have its structure solved at high resolution and remains one the bestunderstood members of this family [2].

Uniquely among vertebrates, the Actinopterygii (rayfinned fish) have not one but two quite distinct rod opsin genes. In this class, the true orthologue of the rod opsin gene found in other vertebrates (including mammals), is not actually expressed in the retina, but rather in the photosensitive pineal gland. The rod opsin found in retinal photoreceptors of ray-finned fish is instead encoded by an intronless gene [3] thought to have arisen by retrotransposition [4]. Because the retinally expressed rod opsin was the first to be described in Actinopterygii, it was termed 'rod opsin' while the subsequently described pineal-specific version was called exo-rhodopsin (exo-rh) or extraretinal rod-like opsin (hereafter termed exo-rod opsin) $[5,6]$. The two rod-like opsins share $\sim 75 \%$ amino acid identity.

Duplication of the Actinopterygian rod opsin gene appears to have been an evolutionarily ancient event. The intronless retinal rod opsin gene appears in basal representatives of this order including bowfin, gar, and sturgeon [7] as well as in the advanced Teleostei [3], but is absent in an extant sarcopterygian fish, the coelacanth Latimeria chalumnae [8]. This places the first appearance of separate rod and exo-rod opsin genes somewhere between the separation of Sarcopterygii and Actinopterygii, 416 million years ago (MYA) [9], and divergence of the neopterygian crown-group (bowfin, gar, and teleosts) at least 284 MYA [10].

Thus, the ray-finned fish have had two separate rod opsin genes for hundreds of millions of years. Assuming that through much of this time one was expressed in the retina and the other in the pineal, significant specialization to the differing demands of photoreception in these two organs might be expected. On this basis, we set out here to undertake the first comprehensive comparative biochemical analysis of rod and exo-rod photopigments. We find that, despite their evolutionary heritage, in at least one important respect (life-time of the principal signaling photoproduct Meta II), exo-rod opsin pigments from zebrafish (Danio rerio) and Fugu pufferfish (Takifugu rubripes) have a functional characteristic more typical of cone opsins. As the amino acid residues responsible for this characteristic in cone opsins are 'rod-like' in exo-rod opsins, these data indicate that exo-rod opsins have attained a cone-like characteristic by a quite different structural mechanism in a novel example of convergent evolution.

\section{Materials and methods}

\section{D4-tagging of opsins}

PCR primers were designed to amplify the coding sequences of the Danio and Fugu rod opsin and exo-rod opsin such that the $5^{\prime}$ end contained a standardized Kozak consensusGCCACCATG [11] and the stop-codon was substituted with an in-frame NheI site (GCTAGC). The Fugu rod and exo-rod opsin coding sequences were amplified from clones 2716 (GenBank: AF201471) and 16h22 (GenBank: AF201472) previously described [5], using the following primer pairs: Fugu Rod 5' Xho I F1, 5'-CCGCTCGAGGCCACCATG AACGGCACGGAGGGACC- $3^{\prime}$ and $F u g u$ Rod $3^{\prime}$ Nhe I R1, 5'-CCGCTAGCCGCAGGAGACACAGAACTGGAGGAGAC-3'; Fugu Exo-Rod 5' Xho I F1, 5'-CCGCTCGAGGC CACCATGAACGGCACGGAAGGACC- $3^{\prime}$ and Fugu ExoRod $3^{\prime}$ Nhe I R1, 5'-CCGCTAGCGGCGGGGGCCACCTG GCTGGAGGAGAC- $3^{\prime}$. The Danio rod and exo-rod opsin coding sequences were amplified from the retinal and pineal cDNA using the following primer pairs: dRod BamHI Kozak F1, 5'-CGGATCCGCCACCATGAACGGTACAGAGGG ACCGGCATTC- $3^{\prime}$ and dRod NheI NoStop R1, 5'-CGCT AGCCGCCGGAGACACGGAGCTGGAAGAC-3'; dExoRod BamHI Kozak F1, 5'-CGGATCCGCCACCATGA ACGGGACGGAGGGACCCAACTTC- $3^{\prime}$ and dExo-Rod NheI NoStop R1, 5'-CGCTAGCGGCTGGAGACACCTG AGCGGAGGAC- ${ }^{\prime}$. The amplified Danio exo-rod and rod opsin sequences are consistent with previous reports, respectively [6] (GenBank: AB025312) and [12] (GenBank: AB187811). A vector (pBluescript II) containing a bovine rod opsin 1D4-tag in the following context, NheI-TETSQVAPAstop (kindly provided by T. Warne, MRC Laboratory of Molecular Biology, Cambridge) allowed in-frame 1D4-tagging of sequence verified opsin coding sequences.

Opsin protein expression

The Flp-In ${ }^{\text {TM }}$ system (Invitrogen) has been used previously for inducible rod opsin expression [13]. The Fugu and Danio 1D4-tagged rod and exo-rod opsins were cloned into 
pcDNA5/FRT/TO and then co-transfected with a pOG44 into Flp-In ${ }^{\mathrm{TM}}-293$ cells. Isogenic stable cell lines were selected with hygromycin at $100 \mu \mathrm{g} / \mathrm{ml}$. Flp-In ${ }^{\mathrm{TM}}$-opsin1D4 cell lines were maintained at $37^{\circ} \mathrm{C}$ in Dulbecco's modified Eagle's medium, 4,500 mg/l D-Glucose, sodium pyruvate and L-glutamine (Sigma) with $10 \%$ fetal bovine serum (Sigma), $10 \mu \mathrm{g} / \mathrm{ml}$ blasticidin and $100 \mu \mathrm{g} / \mathrm{ml}$ hygromycin in a 5\% $\mathrm{CO}_{2}$ atmosphere. Cells were grown in HYPERflasks (Corning) prior to opsin protein expression induction by the presence of $1 \mu \mathrm{g} / \mathrm{ml}$ tetracycline and $5 \mathrm{mM}$ sodium butyrate (B5887, Sigma). Cells were harvested between 20 and $25 \mathrm{~h}$ after induction of expression.

\section{Protein purification}

Cell pellets were re-suspended in PBS, $\mathrm{pH} 7.0$ in the presence of Complete Protease Inhibitors (Roche) and incubated with $50 \mu \mathrm{M} 11$-cis retinal for $2 \mathrm{~h}$ at $4^{\circ} \mathrm{C}$. The cells were subsequently solubilized with $1 \% \beta$-D-dodecylmaltoside (DDM) for $1 \mathrm{~h}$ at $4^{\circ} \mathrm{C}$. Nuclei were pelleted by centrifugation at $21,500 \times g$ and the supernatant was then incubated with 1D4 antibody coupled to $\mathrm{CnBr}$-activated Sepharose (GE Healthcare). After $3 \mathrm{~h}$ rotating at $4^{\circ} \mathrm{C}$, the 1D4 resin was washed with PBS (pH 6), 0.1\% DDM followed by $2 \mathrm{mM}$ sodium phosphate (pH 6), 0.02\% DDM. The purified protein was eluted with the 1D4 peptide TETSQVAPA ( $160 \mu \mathrm{M}$ in $2 \mathrm{mM}$ sodium phosphate $(\mathrm{pH}$ $6), 0.02 \% \mathrm{DDM})$. All procedures after reconstitution were performed under dim red light.

\section{UV-visible spectrophotometry}

Absorption spectra of the purified pigment samples were recorded on a Shimadzu UV-2450 dual-beam spectrophotometer. All spectra were recorded in the $250-750 \mathrm{~nm}$ range. For photo-bleaching experiments, the samples were illuminated with $+515 \mathrm{~nm}$ light for $5 \mathrm{~min}$ and spectra were then recorded. Difference spectra were subsequently calculated. The $\lambda_{\max }$ of each pigment was determined by fitting a standard $\mathrm{A}_{1}$ visual pigment template [14] to the difference spectra using the Solver add-in in Microsoft Excel to generate best-fit curves. This template was kindly provided by D.M. Hunt (Institute of Ophthalmology, University College London).

Chromophore stability of opsins in the dark

The thermal bleaching of the Fugu rod and exo-rod pigments were measured at selected temperatures by the decrease in absorption at $500 \mathrm{~nm}$ [15]. Samples were incubated in the dark for $30 \mathrm{~min}$ at temperatures between 20 and $60^{\circ} \mathrm{C}$ on a gradient thermocycler. After $30 \mathrm{~min}$, the spectra of the samples were re-measured. The amount of remaining pigment with bound chromophore was determined by measurement of the absorption at $500 \mathrm{~nm}$, normalized to the protein absorption at $280 \mathrm{~nm}$. Prism 4.0 ( http://www.graphpad.com) was used to fit sigmoidal curves to the data.

Fluorescent thermal stability assay

A fluorescent dye, the thiol-specific fluorochrome $\mathrm{N}$-[4-(7diethylamino-4-methyl-3-coumarinyl)phenyl]maleimide (CPM) which fluoresces when bound to cysteine residues, was used to assay the thermal stability of the Fugu rod and exo-rod photopigments [16]. Pre-cooled cuvettes were prepared, containing $10 \mu \mathrm{g}$ of opsin protein in a total volume of $110 \mu \mathrm{l}$ sample buffer with detergent $(10 \mathrm{mM}$ HEPES (pH 7.5), $100 \mathrm{mM} \mathrm{NaCl}, 0.1 \mathrm{mM} \mathrm{MgCl}_{2}$, and $0.03 \%$ DDM). Them, $10 \mu \mathrm{l}$ of freshly prepared CPM buffer (sample buffer with $0.1 \mathrm{mg} / \mathrm{ml} \mathrm{CPM}$ ) was added and resuspended by pipetting. A Varian Eclipse spectrofluorometer was used for the assay, with excitation wavelength set at $387 \mathrm{~nm}( \pm 5 \mathrm{~nm})$ and the emission wavelength at $463 \mathrm{~nm}( \pm 10 \mathrm{~nm})$. Dark state assays (in triplicate) were performed between 10 and $90^{\circ} \mathrm{C}$ and a simple mean calculated before normalization.

\section{G-protein purification from bovine retina}

Wild-type G-protein was obtained from 50 bovine retinae. On ice, following exposure to light, the retinae were resuspended in $150 \mathrm{ml}$ of $47 \%(\mathrm{w} / \mathrm{w})$ sucrose in $20 \mathrm{mM}$ Tris $(\mathrm{pH}$ 7.4), $1 \mathrm{mM} \mathrm{CaCl}_{2}, 2 \mathrm{mM}$ DTT and $100 \mathrm{mM}$ PMSF (buffer A) and broken apart against the edge of a beaker until separation was complete. Following centrifugation at $42,000 \times g$ for 15 min the rod outer-segment (ROS) membranes were aspirated from the supernatant fraction. The ROS membranes were diluted $2 \times$ with approximately $200 \mathrm{ml}$ of buffer A and centrifuged for $20 \mathrm{~min}$ at $19,600 \times g$. The pellets were resuspended in $60 \mathrm{ml}$ of buffer A, placed on top of a discontinuous $25-30 \%$ sucrose gradient and centrifuged at $131,000 \times g$ for $20 \mathrm{~min}$. Piercing an injection needle at the orange ROS interface in the tube allowed collection of the membrane layer, which was then diluted in $80 \mathrm{ml}$ buffer A and spun at 42,000 $\times g$ for $15 \mathrm{~min}$. The resulting orange ROS pellet was resuspended and centrifuged once at $42,000 \times g$ for $10 \mathrm{~min}$ in $10 \mathrm{mM}$ Tris $(\mathrm{pH}$ 7.4), $100 \mathrm{mM} \mathrm{NaCl}, 5 \mathrm{mM} \mathrm{MgCl} 2,2 \mathrm{mM}$ DTT, and $1 \mathrm{mM}$ PMSF. This was then washed and centrifuged twice at $42,000 \times g$ for 15 and 20 min using a low-magnesium buffer 10 mM Tris (pH 7.4), 0.1 mMEDTA, 2 mMDTT and $1 \mathrm{mM}$ PMSF (buffer B). G-protein bound to the lightexposed rod opsin was eluted for $30 \mathrm{~min}$ from resuspended ROS membranes in $25 \mathrm{ml}$ buffer B supplemented with $40 \mu \mathrm{M}$ GTP. ROS membranes were separated from eluted 
G-protein by centrifugation at $257,000 \times g$ for $15 \mathrm{~min}$. Supernatant was allowed to dialyze in $10 \mathrm{mM}$ Tris (pH7.4), $2 \mathrm{mM} \mathrm{MgCl}, 2 \mathrm{mM}$ DTT in $50 \%$ glycerol with three changes in $36 \mathrm{~h}$. Extracted proteins were stored in aliquots at $-20^{\circ} \mathrm{C}$.

\section{G-protein fluorescence GTP $\gamma$ S binding assay}

Opsin interaction with the G-protein transducin $\left(\mathrm{G}_{\mathrm{t}}\right)$ was analyzed by fluorescence spectroscopy, based on an intrinsic increase in tryptophan fluorescence of $G_{t}$ [17]. The assay makes use of the increase in tryptophan fluorescence after uptake of a non-hydrolysable GTP $\gamma \mathrm{S}$ nucleotide by the $\mathrm{G} \alpha$ subunit upon activation. The increase in fluorescence quantum yield of the $\mathrm{G} \alpha(\mathrm{GTP} \gamma \mathrm{S})$ complex is 2.3 times higher than the $\mathrm{G} \alpha(\mathrm{GDP})$ complex [18]. A total of $10 \mathrm{nM}$ of each of the Fugu and Danio rod and exo-rod pigments were photobleached after continuous illumination at 515-nm wavelength of light for $20 \mathrm{~s}$. This was then added to a cuvette containing $250 \mathrm{nM}$ bovine rod transducin $\left(\mathrm{G}_{\mathrm{t}}\right)$ in $10 \mathrm{mM}$ Tris- $\mathrm{HCl}, \mathrm{pH} 7.4,100 \mathrm{mM} \mathrm{NaCl}$, $2 \mathrm{mM} \mathrm{MgCl} 2,0.008 \%$ DDM in a total volume of $1.2 \mathrm{ml}$, and allowed to stir slowly for $100 \mathrm{~s}$. A stock solution of $10 \mathrm{mM} \mathrm{GTP} \gamma \mathrm{S}$ was added to a $5 \mu \mathrm{M}$ final concentration after $10 \mathrm{~s}$. The increase in tryptophan fluorescence at an emission wavelength of $340 \mathrm{~nm}$ was then recorded for an additional 2,500 s in a Beckman LS55 spectrofluorometer. G-protein activation assays were carried out at $14^{\circ} \mathrm{C}$. Bovine rod opsin was used as a comparable positive control in these experiments, and Fugu rod opsin in the absence of GTP $\gamma \mathrm{S}$ provided a negative control.

\section{FTIR spectroscopy}

FTIR spectroscopy was performed with Fugu rod and exorod pigments reconstituted into phosphatidyl choline lipids (from egg yolk). Lipid reconstitution was achieved at $4^{\circ} \mathrm{C}$ by combining the DDM-purified pigments with DDM-solubilized lipid at a molar ratio of 1:200. After incubation for $2 \mathrm{~h}$, DDM was removed by three rounds of extraction using small washed polystyrene beads (with a detergent binding capacity $5 \times$ higher than the initially present DDM; Bio-Beads SM-2, Bio-Rad, Hercules, CA, USA) for $4 \mathrm{~h}$. After careful removal of the Bio-Beads, proteoliposomes were collected by centrifugation for $4 \mathrm{~h}$ at $100,000 \times \mathrm{g}$. Spectra were recorded at $4 \mathrm{~cm}^{-1}$ with a scanning time of $30 \mathrm{~s}$ using a Bruker Vertex 70 FTIR spectrometer equipped with an MCT detector using 100 pmol reconstituted pigment in sandwich samples [19] and $200 \mathrm{mM}$ BTP (at pH 6.0) or MES buffer (at pH 5.0). Meta II spectra of $F u g u$ exo-rod opsin were recorded with a time resolution of $6 \mathrm{~s}$ at $10^{\circ} \mathrm{C}$ to compensate for its faster decay rate. Photoactivation was achieved by a $1 \mathrm{~s}$ photolysis using an array of 6 LEDs at $530 \mathrm{~nm}$ [20]. Meta II decay was monitored by following the decrease of intensity of bands in the range between 1,800 and $1,600 \mathrm{~cm}^{-1}$ and at $1,570 \mathrm{~cm}^{-1}$.

\section{Results}

Spectral sensitivity of the Fugu and Danio opsins

Our first step in comparing the functional characteristics of rod and exo-rod opsin pigments was to determine their spectral absorbance properties in the UV-visible range. Microspectrophotometry of retinal and pineal photoreceptors in some deep-sea species suggest significant differences in spectral sensitivity [21], but no direct comparison of this parameter between rod and exo-rod opsin proteins has been reported. To address this deficit, rod and exo-rod opsin proteins from zebrafish (Danio rerio) and Fugu pufferfish (Takifugu rubripes), were expressed in HEK293 cells, reconstituted with the $\mathrm{A}_{1}$ chromophore 11-cis retinaldehyde, solubilized in dodecylmaltoside, and 1D4 immunoaffinity purified. This process provided good yields of all four proteins. UV-vis spectroscopy revealed significant absorbance in the visible range for each pigment (Fig. 1). This visible absorption was lost in favor of enhanced UV absorption following bright light exposure, suggesting that all four pigments could be photobleached. In order to define their spectral sensitivity, dark-bleach difference spectra were plotted (Fig. 1 insets) and fitted with the Govardovskii et al. [14] opsin absorbance template. These revealed very similar $\lambda_{\max }$ across all four opsins at $501.8 \mathrm{~nm}$ (Danio rod; $\left.\mathrm{SS}^{2}=0.0000, R^{2}=0.9950\right), 498.2 \mathrm{~nm}$ (Danio exo-rod; $\left.\mathrm{SS}^{2}=0.0000, R^{2}=0.9967\right), 501.4 \mathrm{~nm}\left(\right.$ Fugu rod; $\mathrm{SS}^{2}=$ $\left.0.0000, \quad R^{2}=0.9994\right)$, and $497.8 \mathrm{~nm}$ (Fugu exo-rod; $\left.\mathrm{SS}^{2}=0.0000, R^{2}=0.9957\right)$. The Danio rod opsin $\lambda_{\max }$ is consistent with published in vitro [22] and in vivo [23, 24] reports. The rod opsin from both species possess a $\lambda_{\max }$ $\sim 3.5 \mathrm{~nm}$ longer than the corresponding exo-rod opsin. When comparing the primary structure of the two types of opsin, in both species position 124 is Gly124 in the rod opsin, but Ala124 in the exo-rod opsin. Ala124Gly substitutions are associated with up to $+3 \mathrm{~nm}$ shifts in the $\lambda_{\max }$ of the rod opsins of certain deep-sea fish [25], and it is quite possible that these substitutions account for much of the spectral shift seen between the rod and exo-rod. While the Ser299Ala substitution observed between the Fugu rod and exo-rod pigments could also account for $\sim 2 \mathrm{~nm}$ [26] of the $\lambda_{\max }$ shift, as yet undetermined spectral tuning residues and their synergistic effects regarding $\lambda_{\max }$ exist for rod (RH1) opsins $[25,27]$. 
Fig. 1 Absorption spectra of rod and exo-rod opsins. $A_{1}$ reconstituted and purified rod and exo-rod pigments from Danio and Fugu measured in the dark (black line) and after photobleaching (grey line). The $\lambda_{\max }$ calculated by fitting the Govardovskii et al. [14] template to the dark-light difference spectra (insets) are indicated

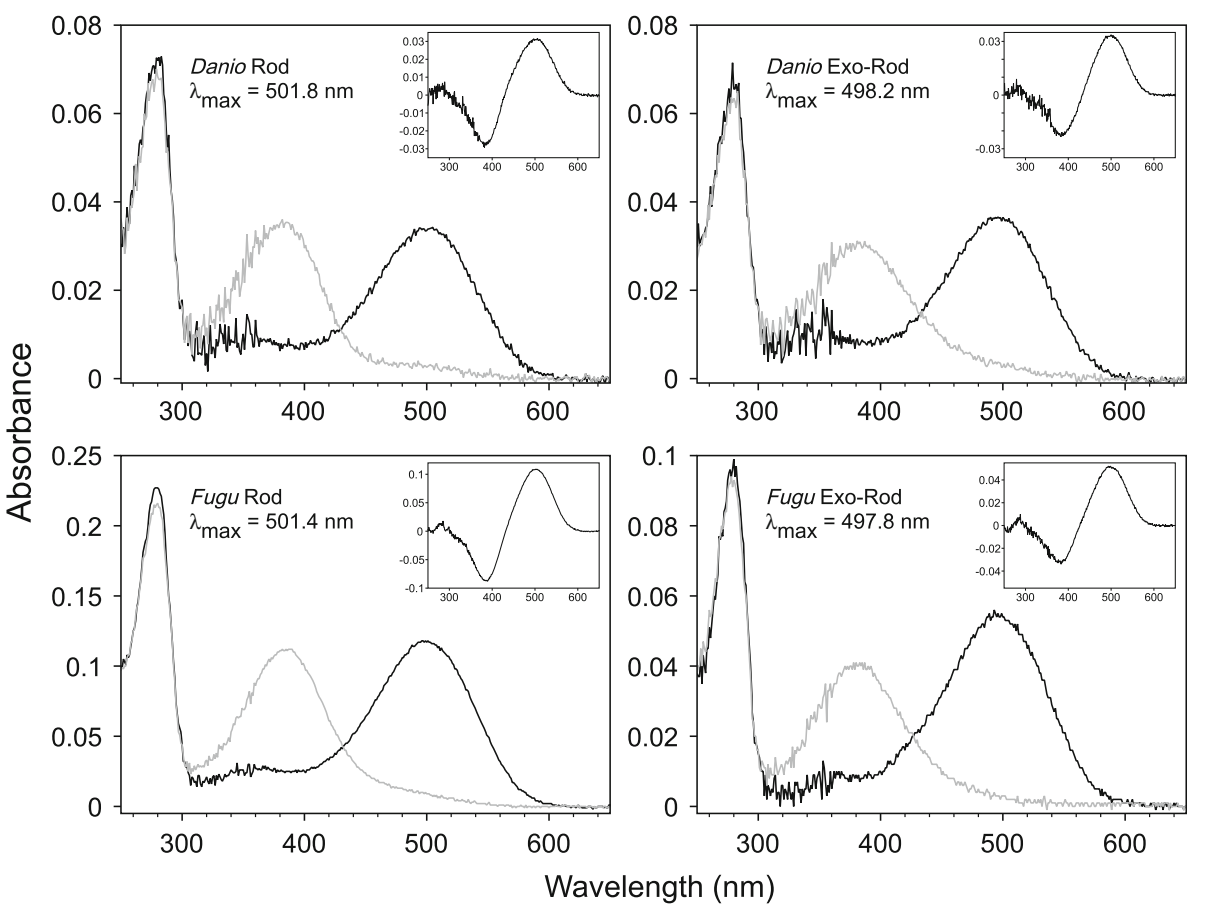

Thermal bleach

Given the similarity of the spectral sensitivities of rod and exo-rod opsin pigments, we set out to explore other biochemical parameters in which the two opsins might differ. Opsin photopigments spontaneously bleach in the dark due to thermal isomerization of the chromophore and/or spontaneous hydrolysis of the Schiff base linkage [28]. This occurs at a much higher rate in cone than in rod opsins [29, 30]. As free retinaldehyde absorbs UV light, thermal bleach can be traced in the same way as light bleach, by the reduction in absorbance at visible wavelengths. We used this feature to compare the thermal release of chromophore by the Fugu rod and exo-rod photopigments. We observed a temperature-dependent reduction in visible absorbance in both pigments (Fig. 2a). Incubation for $30 \mathrm{~min}$ at $<41^{\circ} \mathrm{C}$ had little effect on either Fugu rod or exo-rod photopigments, but incubation at higher temperatures caused progressive decreases in the amplitude of the visible absorption peak until, by $60^{\circ} \mathrm{C}$, neither pigment showed any detectable absorption around $500 \mathrm{~nm}$. To compare the temperature dependence of this dark bleach, we plotted the change in absorbance at $500 \mathrm{~nm}$ as a function of temperature for each pigment. This revealed that, while the Fugu exo-rod opsin was bleached significantly at temperatures between 44.5 and $48.7^{\circ} \mathrm{C}$, equivalent reductions in $F u g u$ rod absorption were not recorded until $48.7-53.8^{\circ} \mathrm{C}$. The temperature at which $50 \%$ of the bound retinal is lost $\left(T_{50}\right)$ was calculated as $52.3^{\circ} \mathrm{C}$ for $F u g u$ rod opsin and $47.1^{\circ} \mathrm{C}$ for Fugu exo-rod opsin.
We next turned to the thermal stability of the opsin protein itself using CPM, a thiol-specific fluorochrome [16]. This fluorochrome fluoresces when covalently bound to cysteines, but not when free in solution. As such an attachment requires (partial) unfolding of the protein, its fluorescence can be used to analyze the thermal integrity of detergent solubilized membrane proteins. Thermal denaturation scans generated curves whose shape mirrored those of chromophore release (Fig. 2b), enabling thermal 'half-melting' temperatures $\left(\mathrm{Tm}_{50}\right)$ of $60.4^{\circ} \mathrm{C}$ for $\mathrm{Fugu} \operatorname{rod}$ opsin and $56.3^{\circ} \mathrm{C}$ for $\mathrm{Fugu}$ exo-rod opsin to be calculated.

\section{G-protein activation}

The G-protein activation ability of purified Fugu and Danio photopigments was examined using bovine transducin $\left(G_{t}\right)$ in a standard intrinsic tryptophan fluorescence assay [17] employing 1D4 purified Fugu and Danio rod and exo-rod opsins in dodecylmaltoside (DDM) detergent solution. The rod opsins and exo-rod opsins from both teleost species were able to activate heterotrimeric G-protein (Fig. 3). The teleost rod photopigments performed nearly as effectively as bovine rod rhodopsin in this assay, with final totals of G-protein turnover $81.0 \%$ (Fugu) and $85.1 \%$ (Danio) those of bovine rod rhodopsin. By contrast, both exo-rod opsins showed much lower total relative G-protein activation at 28.1 and $26.0 \%$ that of the bovine rod rhodopsin for Fugu and Danio respectively. 

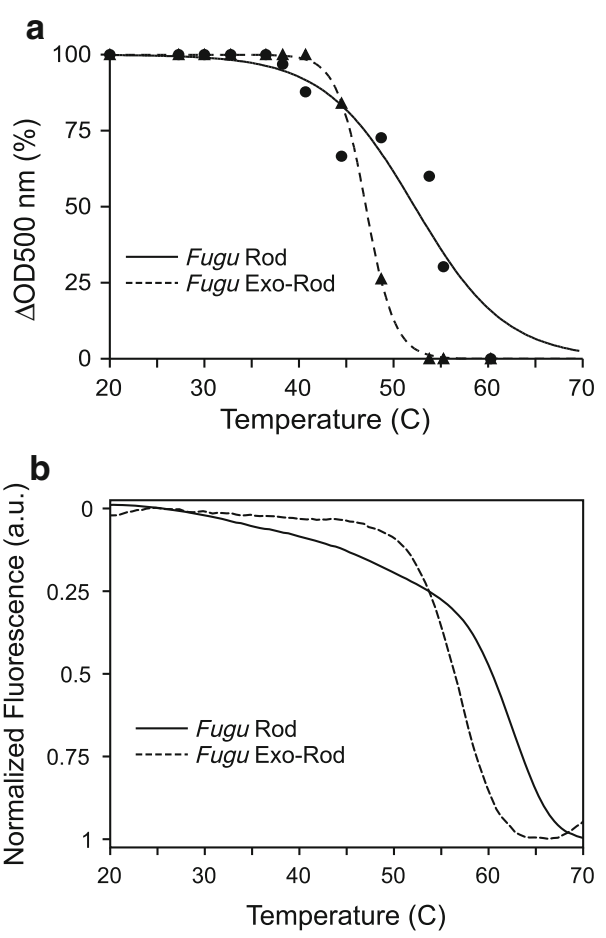

Fig. 2 Thermal stability of the Fugu pigments in the dark state. a Chromophore loss as a function of temperature. The percentage change in absorbance at $500 \mathrm{~nm}$ was plotted against temperature as a measure of the thermal release of chromophore. Sigmoidal curves fitted to the data points using the program Prism 4.0 allowed $T_{50}$ values to be determined-Fugu rod $T_{50}=52.3^{\circ} \mathrm{C}$ and $F u g u$ exo-rod $T_{50}=47.1^{\circ} \mathrm{C}$. (Filled circles, Fugu rod; filled triangles, Fugu exorod). b Thermal denaturation curves for Fugu rod and exo-rod pigments determined by CPM dye assay. Curves are the average of three experiments subjected to least-square smoothing and normalization in Plot 0.997 , and the interpolated 50\% 'melt' values are $T m_{50}=60.4^{\circ} \mathrm{C}$ for Fugu rod opsin, and $T m_{50}=56.3^{\circ} \mathrm{C}$ for $F u g u$ exo-rod

FTIR difference spectroscopy of Fugu pigment photoproducts

We then used light-induced FTIR difference spectroscopy to obtain more insight into structural and activation differences between the two Fugu pigments. The photopigments were reconstituted into egg PC lipid membranes and were compared to similarly lipid-reconstituted bovine rod rhodopsin under otherwise identical conditions. Difference spectra Meta II minus dark state (Fig. 4a) revealed band patterns containing, among others, difference bands of the $\mathrm{C}=\mathrm{O}$ stretch of protonated carboxylic acids (above $1,700 \mathrm{~cm}^{-1}$ ), participating both in interhelical hydrogen bonded networks and in proton transfer reactions, or of the protein backbone in the amide I range (around $1,650 \mathrm{~cm}^{-1}$ ).

The difference spectra of both Fugu pigments revealed a positive band at $1,712 \mathrm{~cm}^{-1}$ similar to that of bovine rod opsin, reflecting protonation of Glu113 by proton transfer

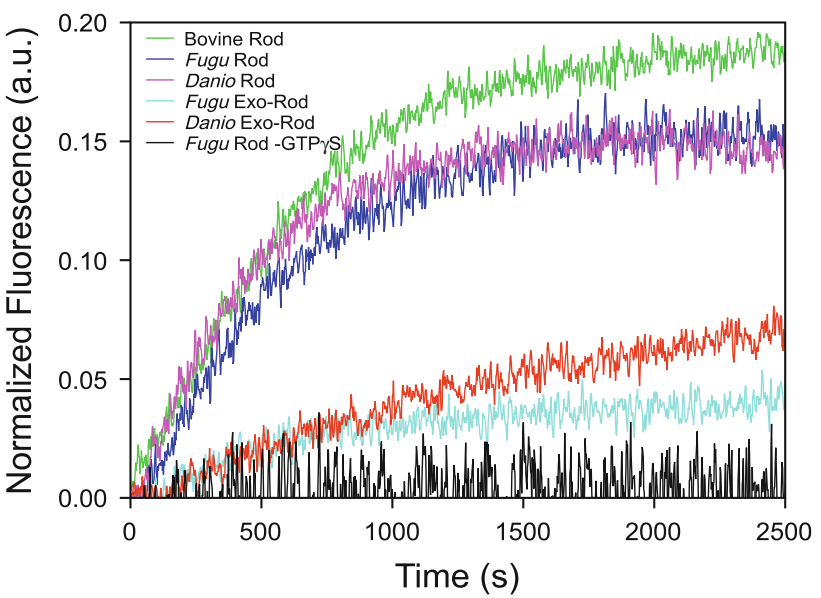

Fig. 3 Transducin activation by photoactivated opsins. Fugu and Danio photoproduct interactions with transducin $\left(\mathrm{G}_{\mathrm{t}}\right)$ were compared to those of bovine rod opsin using a fluorescent GTP $\gamma \mathrm{S}$ binding assay. Total G-protein activation relative to that by bovine rod photoproduct was calculated by integration of each curve. A control reaction in the absence of GTP $\gamma \mathrm{S}$ was also undertaken for Fugu rod photoproduct (Fugu rod-GTP $\gamma \mathrm{S}$ )

from the PSB [31] and of Glu134 by proton uptake from the solvent in Meta II [32]. This indicates a similar triggering of these two protonation switches of the activation process as in rod opsin [20]. In Fugu rod Meta II, this band was superimposed upon an additional band at $1,702 \mathrm{~cm}^{-1}$ that is not assigned.

The difference band at $1,728 \mathrm{~cm}^{-1}(-) / 1,747 \mathrm{~cm}^{-1}(+)$ in rod opsin is attributed to changes of hydrogen bonding of Glu122 close to the ring of retinal, which participates in an interhelical network between $\mathrm{H} 3$ and $\mathrm{H} 5$ involving His 211 and Trp126 and which is a key residue in the decay of the signaling state of rod and cone pigments [33]. Similar to bovine rod opsin, both Fugu pigments reveal a downshift of this band, corresponding to a decrease of the hydrogen bonding of this residue. In addition, the intensity of its positive lobe at $1,747 \mathrm{~cm}^{-1}$ is reduced in Fugu exorod opsin Meta II, indicating an altered environment of Glu122 as compared with bovine rod opsin Meta II or Fugu rod Meta II.

Finally, the environment of Asp83 of a conserved watermediated network between $\mathrm{H} 1, \mathrm{H} 2$, and $\mathrm{H} 7$ can be monitored using its difference band at $1,768 \mathrm{~cm}^{-1}$ $(-) / 1,747 \mathrm{~cm}^{-1}(+)$ (its positive lobe overlapping with that of Glu122). Both in Fugu rod and (to a slightly lesser extent) in Fugu exo-rod opsin the dark absorption of Asp83 is downshifted from its position at $1,768 \mathrm{~cm}^{-1}$ in bovine rod opsin, leading to an overall reduction of the difference band. This alteration of the interhelical network in the dark state is possibly induced by substitutions of neighboring Ser298Ala299 to Ser298Ser299 in Fugu rod and Ala298Ala299 in Fugu exo-rod opsin, of which residue 299 is a known $\pm 2 \mathrm{~nm}$ spectral tuning site [26]. 

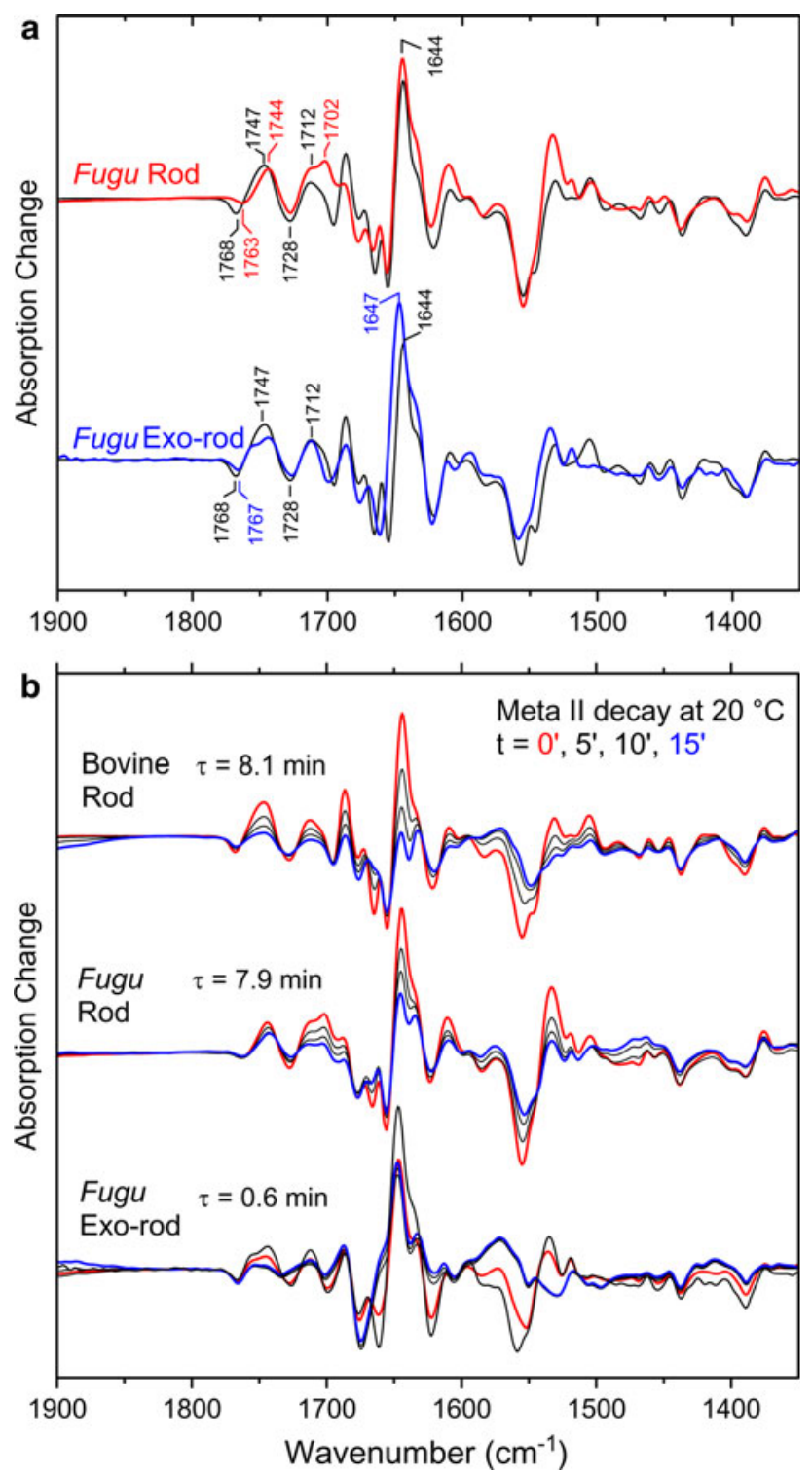

Fig. 4 FTIR spectroscopy on formation and decay of Meta II in Fugu pigments. a Light-induced FTIR difference spectra Meta II minus dark state were obtained from Fugu rod (red spectra) and exo-rod (blue spectra) pigments reconstituted into lipid membranes at $20^{\circ} \mathrm{C}, \mathrm{pH} 6.0$, and $10^{\circ} \mathrm{C}, \mathrm{pH} 5.0$, respectively, and are compared to bovine rod rhodopsin (black spectra) under otherwise identical conditions. The spectra reveal the general conformational changes observed for activation of bovine rod rhodopsin with smaller pigment-specific alterations discussed in the text. b The decay of Meta II to opsin and free all-trans retinal was followed at $20^{\circ} \mathrm{C}, \mathrm{pH} 6.0$, in successive spectra of 0.5 min sampling time starting at the indicated time after photoactivation. These spectra reveal a similar time constant for the decay of Fugu rod Meta II as for bovine rod opsin Meta II of roughly 8 min, while the decay of Fugu exo-rod Meta II is more than one order of magnitude faster. As there was already substantial decay of Meta II in the first spectrum after photoactivation of Fugu exo-rod, the full exo-rod Meta II spectrum obtained at $10^{\circ} \mathrm{C}$ is shown with a dotted line

The thermal decay of the Meta II state to opsin and free all-trans retinal was followed by time-resolved spectroscopy at $20^{\circ} \mathrm{C}, \mathrm{pH} 6.0$, using the conformationally sensitive bands in the range between 1,600 and $1,800 \mathrm{~cm}^{-1}$ and bands of retinal at around $1,570 \mathrm{~cm}^{-1}$ (Fig. 4b) [19]. Under these conditions, no significant decay to Meta III [34] nor structural instability of the pigment [35] were observed. While Fugu rod and bovine rod Meta II decayed with a similar time constant ( $\tau=\sim 8 \mathrm{~min})$, Fugu exo-rod opsin Meta II decay was accelerated by more than an order of magnitude $(\tau=0.6 \mathrm{~min})$. The identity of the decay product with opsin was confirmed by comparison with opsin generated by illumination in the presence of hydroxylamine (Supplementary Material Fig. 1). The Meta II decay of Danio exo-rod opsin exhibits a similar time constant $(\tau=\sim 0.5 \mathrm{~min}$ ) to that of Fugu exo-rod opsin.

\section{Discussion}

To our knowledge, these data represent the first biochemical characterization of exo-rod opsin pigments. We show that while Danio and Fugu exo-rod opsins have spectral sensitivities within the range expected for rod opsins $\left(\lambda_{\max }\right.$ around $500 \mathrm{~nm}$ ), in other respects their behavior is quite atypical for the rod opsin family.

Of all the differences that we have observed in this study between the rod and exo-rod pigments, the least distinct is that in spectral sensitivity. The native habitat for Danio is freshwater pools and streams [36], while that of Fugu is of coastal marine waters to a maximal depth of $150 \mathrm{~m}$ [37]. Vertebrates residing in such environments typically possess a rod with $\lambda_{\max } \sim 500 \mathrm{~nm}$ [38], and our description of the $A_{1}$ spectral sensitivities of rod opsins from these two species matches this prediction. In both of these species, we find that the exo-rod pigment is blue shifted by $\sim 3.5 \mathrm{~nm}$, with the $\sim 498 \mathrm{~nm} \lambda_{\max }$ that we have determined for $A_{1}$ Danio exo-rod being consistent with a prediction based on its primary structure [6], and with the spectral sensitivity of the major peak for light induced pineal melatonin suppression in this species at $\sim 500 \mathrm{~nm}$ [39]. Recently, one of the likely signaling consequences of exo-rod opsin in the zebrafish pineal has been shown to be the regulation of expression of aanat2 [40], which encodes the pineal specific version of arylalkylamine $N$-acetyltransferase [41], the penultimate enzyme in the synthetic pathway for melatonin.

Even though the $\lambda_{\max }$ of the $\mathrm{A}_{1}$ rod and exo-rod pigments in this study are very similar, this observation appears not to hold for all teleost species since microspectrophotometric evidence from both marine and freshwater teleosts indicates that opsin moiety spectral differences exist between the $\lambda_{\max }$ of retinal rod and presumed exo-rod pineal photoreceptors [21, 42]. In addition freshwater fish can exhibit substitution or co-utilization of the $\mathrm{A}_{1}$ and porphyropsin-forming $\mathrm{A}_{2}$ chromophore, 11-cis 
3-dehydroretinal [43], which will red-shift the $\lambda_{\max }$ of both the rod and exo-rod pigment [42]. Thus, both opsin tuning and chromophore usage could provide scope for significant differences in the in vivo spectral sensitivity of the rod and pineal photoreceptors.

Among the cardinal biochemical features of retinal rod photopigments is their low rate of spontaneous (thermal) activation in the dark [44], e.g., salamander rods have an estimated spontaneous activation of $\sim 0.03 \mathrm{~s}^{-1}$ [45] whereas $\mathrm{L}$ cones experience a rate of $\sim 600 \mathrm{~s}^{-1}$ [29], which when corrected for pigment density suggests that the cone pigment is $6 \times 10^{5}$ times less stable than the rod pigment [29]. Correspondingly, expression of cone opsins in rod photoreceptors results in low-level activation of phototransduction in the dark, precluding full dark adaptation and reducing sensitivity [30,46]. This low level of spontaneous rod opsin activation is thought to be critical for the extremely high sensitivity of rod photoreceptors [47], and can be attributed to the ability of the opsin moiety to retain the 11-cis-retinal chromophore that acts as an inverse agonist suppressing activation of the receptor [48]. We show here that at physiological temperatures both Fugu rod and exo-rod pigments show roughly similar levels of thermal stability and it is only at higher temperatures do differences in stability manifest themselves. This physiological stability is consistent with that previously observed for rod opsin [49], and contrasts markedly with the stability of cone opsins [50]. The relative stability of the pineal photopigment is consistent with the observation that the estimated absolute sensitivity of pineal photoreceptors is comparable to those of retinal rods [51]. This is perhaps not unexpected given the anatomical position of the pineal and the associated filtering effects of the skull and associated tissues, light intensities experienced by the pineal in situ are typically between 1/10th and 1/100th that of ambient and enriched for longer wavelengths [52-55]. Note, however, the rate at which photoreceptors experience spontaneous activation of the phototransduction cascade in the dark would depend not only on the rate of thermal bleach but also on the total quantity of pigment. Although rod and pineal photopigment density does seem to be comparable with similar optical densities reported by microspectrophotometry on rod and pineal photoreceptors in trout [56], the pineal photoreceptors lack the extensive pigment-dense outer segment discs found in retinal rods (typically in excess of 1,000) and have 20-70 lamellae [57], with correspondingly low levels of chromophoreconcentrations being $1 / 300-1 / 1,000$ that of retinal photoreceptors [58].

Another important difference between rod and cone pigments is the lifetime of their light-activated state. Following light absorption both opsin classes progress through a series of meta-stable photoproduct states, including the
Meta II signaling state capable of activating the G protein [1]. However, rod signal transduction is characterized by a much longer lifetime of the Meta II product than in cone pigments [59], with Meta II time constants of $\sim 480 \mathrm{~s}$ ( $\sim 8 \mathrm{~min})$ for rods and $\sim 5 \mathrm{~s}(\sim 0.12 \mathrm{~min})$ for cones [60]. In vitro assays indicate that this difference between the Meta II lifetime of rod and cone pigments results in significantly reduced G-protein activation by cone opsins compared to rod opsin [61]. Here, we found an equivalent discrepancy in G-protein activation between rod and exorod opsin pigments. In theory, the reduced activity of exorod opsin could reflect lower affinity for the G-protein provided (bovine transducin). However, this seems unlikely given the high sequence similarity between exo-rod opsin and bovine rod opsin in G-protein interaction domains (predicted intracellular loops 2 and 3 show 91 and $67 \%$ identity; for reference these figures are 82 and $62 \%$ for Fugu rod opsin), and the fact that the Fugu genome has only a single rod transducin alpha-subunit (GNAT1) [62], which is presumably used by both rod opsin and exo-rod opsin pigments. However, given that both rod and cone transducins are expressed in the teleost pineal [63], we cannot exclude the possibility that exo-rod opsin interacts with the cone transducin alpha-subunit (GNAT2). Recent evidence from transgenic mice indicates that Gnat2 will interact with rod opsin leading to rods that exhibit responses with a decreased sensitivity and rate of activation half that of Gnat1 [64]. The equivalent sensitivity of the pineal and rod photoreceptors would suggest that exo-rod opsin is interacting with a GNAT1 rather than a GNAT2 subunit. The alternative explanation, that it reflects lower lifetime of signaling photoproducts, is supported by our FTIR analysis. Thus, time-resolved FTIR spectroscopy reveals that the Meta II lifetime of Fugu exo-rod opsin is reduced by about one order of magnitude compared with Fugu or bovine rod opsins. In vivo, the reduction in Meta II lifetime for cone opsins may facilitate bleach recovery, allowing cones to function under continuous bright illumination. The reduction in Meta II lifetime of exo-rod opsin could perform a similar function for teleost pineal photoreceptors, with a fast bleach recovery allowing them to maintain a high absolute sensitivity while having a low level of photopigment. Thus, the reduced Meta II lifetime of exo-rod opsin could contribute to the observation that pineal photoreceptors are active over a greater range of light intensities than retinal photoreceptors [57], e.g., from physiological recordings it has been estimated that the dynamic range of pineal photoreceptors can be 2-3 times larger than their retinal counterparts [65].

Interestingly, the decreased Meta II lifetime of exo-rod opsin pigments are not reflected in a 'cone-like' primary structure. Site-directed mutagenesis has revealed the residues at positions 122 and 189 , which interact with the 
Table 1 Relative Meta II decay rates of photopigments in relation to the amino acid content of positions 122 and 189

Note that the rod photopigment Meta II decay rates from both studies are deemed to be equivalent and normalized to 1

a This study

b [66]

\begin{tabular}{llll}
\hline Photopigment & $\begin{array}{l}\text { Relative Meta II } \\
\text { decay rate }\end{array}$ & $\begin{array}{l}\text { Residue } \\
122\end{array}$ & $\begin{array}{l}\text { Residue } \\
189\end{array}$ \\
\hline Bovine rod & $1^{\mathrm{a}}$ & Glu & Ile \\
Chicken rod (wild-type) & $1^{\mathrm{b}}$ & Glu & Ile \\
Chicken rod (double mutant) & $22^{\mathrm{b}}$ & Gln & Pro \\
Fugu exo-rod & $13^{\mathrm{a}}$ & Glu & Ile \\
Danio exo-rod & $16^{\mathrm{a}}$ & Glu & Ile \\
Fugu rod & $1^{\mathrm{a}}$ & $\mathrm{Glu}$ & Val \\
Chicken green cone (wild-type) & $70^{\mathrm{b}}$ & Gln & Pro \\
Chicken green cone (double mutant) & $0.63^{\mathrm{b}}$ & Glu & Ile \\
\hline
\end{tabular}

positions 122 and 189 has implications for the evolution of these photopigments. Current evidence suggests that rod opsin evolved from a cone opsin [68, 69]. Thus, one potential explanation for the cone-like characteristic of the exo-rod opsin pigments is that they simply reflect those of the ancestral pigment. In this view, it would be the retinal rod opsin whose characteristics had evolved to match its sensory function. In fact, the structural basis for accelerated Meta II decay appears quite different between the cone and exo-rod opsin pigments (see above). Thus it seems that the ancestor of both Actinopterygian rod proteins had classical rod-like functional characteristics, and it has been the exorod opsin that has diverged to attain a cone-like characteristic by convergent evolution.

Acknowledgments This work was supported by funding from The Wellcome Trust to RJL, JB, GFXS, and MWH (grant numbers 078808/A/05/Z, 078808/B/05/Z); Deutsche Forschungsgemeinschaft to RV (grant number VO 811/4-1); and additional support from the National Institute for Health Research Manchester Biomedical Research Centre and the Manchester Academic Health Science Centre was provided to JB.

Open Access This article is distributed under the terms of the Creative Commons Attribution Noncommercial License which permits any noncommercial use, distribution, and reproduction in any medium, provided the original author(s) and source are credited.

\section{References}

1. Menon ST, Han M, Sakmar TP (2001) Rhodopsin: structural basis of molecular physiology. Physiol Rev 81:1659-1688

2. Schertler GFX (2005) Structure of rhodopsin and the metarhodopsin I photointermediate. Curr Opin Struct Biol 15:408-415

3. Fitzgibbon J, Hope A, Slobodyanyuk SJ, Bellingham J, Bowmaker JK, Hunt DM (1995) The rhodopsin-encoding gene of bony fish lacks introns. Gene 164:273-277

4. Bellingham J, Tarttelin EE, Foster RG, Wells DJ (2003) Structure and evolution of the teleost extraretinal rod-like opsin (errlo) and ocular rod opsin (rho) genes: is teleost rho a retrogene? J Exp Zool B Mol Dev Evol 297:1-10

5. Philp AR, Bellingham J, Garcia-Fernandez J-M, Foster RG (2000) A novel rod-like opsin isolated from the extra-retinal photoreceptors of teleost fish. FEBS Lett 468:181-188 
6. Mano H, Kojima D, Fukada Y (1999) Exo-rhodopsin: a novel rhodopsin expressed in the zebrafish pineal gland. Brain Res Mol Brain Res 73:110-118

7. Venkatesh B, Ning Y, Brenner S (1999) Late changes in spliceosomal introns define clades in vertebrate evolution. Proc Natl Acad Sci USA 96:10267-10271

8. Yokoyama S, Zhang H, Radlwimmer FB, Blow NS (1999) Adaptive evolution of color vision of the Comoran coelacanth (Latimeria chalumnae). Proc Natl Acad Sci USA 96:6279-6284

9. Benton MJ, Donoghue PC (2007) Paleontological evidence to date the tree of life. Mol Biol Evol 24:26-53

10. Hurley IA, Mueller RL, Dunn KA, Schmidt EJ, Friedman M, Ho RK, Prince VE, Yang Z, Thomas MG, Coates MI (2007) A new time-scale for ray-finned fish evolution. Proc R Soc Lond B Biol Sci 274:489-498

11. Xia $X$ (2007) The $+4 \mathrm{G}$ site in Kozak consensus is not related to the efficiency of translation initiation. PLoS One 2:e188

12. Hamaoka T, Takechi M, Chinen A, Nishiwaki Y, Kawamura S (2002) Visualization of rod photoreceptor development using GFP-transgenic zebrafish. Genesis 34:215-220

13. Noorwez SM, Kuksa V, Imanishi Y, Zhu L, Filipek S, Palczewski K, Kaushal S (2003) Pharmacological chaperone-mediated in vivo folding and stabilization of the $\mathrm{P} 23 \mathrm{H}$-opsin mutant associated with autosomal dominant retinitis pigmentosa. J Biol Chem 278:14442-14450

14. Govardovskii VI, Fyhrquist N, Reuter T, Kuzmin DG, Donner K (2000) In search of the visual pigment template. Vis Neurosci 17:509-528

15. Standfuss J, Xie G, Edwards PC, Burghammer M, Oprian DD, Schertler GFX (2007) Crystal structure of a thermally stable rhodopsin mutant. J Mol Biol 372:1179-1188

16. Alexandrov AI, Mileni M, Chien EY, Hanson MA, Stevens RC (2008) Microscale fluorescent thermal stability assay for membrane proteins. Structure 16:351-359

17. Ramon E, Marron J, del Valle L, Bosch L, Andrés A, Manyosa J, Garriga P (2003) Effect of dodecyl maltoside detergent on rhodopsin stability and function. Vis Res 43:3055-3061

18. Phillips WJ, Cerione RA (1988) The intrinsic fluorescence of the alpha subunit of transducin. Measurement of receptor-dependent guanine nucleotide exchange. J Biol Chem 263:15498-15505

19. Vogel R, Siebert F (2001) Conformations of the active and inactive states of opsin. J Biol Chem 276:38487-38493

20. Mahalingam M, Martinez-Mayorga K, Brown MF, Vogel $\mathrm{R}$ (2008) Two protonation switches control rhodopsin activation in membranes. Proc Natl Acad Sci USA 105:17795-17800

21. Bowmaker JK, Wagner HJ (2004) Pineal organs of deep-sea fish: photopigments and structure. J Exp Biol 207:2379-2387

22. Chinen A, Hamaoka T, Yamada Y, Kawamura S (2003) Gene duplication and spectral diversification of cone visual pigments of zebrafish. Genetics 163:663-675

23. Nawrocki L, BreMiller R, Streisinger G, Kaplan M (1985) Larval and adult visual pigments of the zebrafish, Brachydanio rerio. Vis Res 25:1569-1576

24. Allison WT, Haimberger TJ, Hawryshyn CW, Temple SE (2004) Visual pigment composition in zebrafish: Evidence for a rhodopsin-porphyropsin interchange system. Vis Neurosci 21:945952

25. Hunt DM, Dulai KS, Partridge JC, Cottrill P, Bowmaker JK (2001) The molecular basis for spectral tuning of rod visual pigments in deep-sea fish. J Exp Biol 204:3333-3344

26. Fasick JI, Robsinson PR (1998) Mechanism of spectral tuning in the dolphin visual pigments. Biochemistry 37:433-438

27. Yokoyama S, Tada T, Zhang H, Britt L (2008) Elucidation of phenotypic adaptations: molecular analyses of dim-light vision proteins in vertebrates. Proc Natl Acad Sci USA 105:13480-13485
28. Ebrey T, Koutalos Y (2001) Vertebrate photoreceptors. Prog Retin Eye Res 20:49-94

29. Rieke F, Baylor DA (2000) Origin and functional impact of dark noise in retinal cones. Neuron 26:181-186

30. Kefalov V, Fu Y, Marsh-Armstrong N, Yau KW (2003) Role of visual pigment properties in rod and cone phototransduction. Nature 425:526-531

31. Jäger F, Fahmy K, Sakmar TP, Siebert F (1994) Identification of glutamic acid 113 as the Schiff base proton acceptor in the metarhodopsin II photointermediate of rhodopsin. Biochemistry 33:10878-10882

32. Vogel R, Mahalingam M, Lüdeke S, Huber T, Siebert F, Sakmar TP (2008) Functional role of the "ionic lock"-an interhelical hydrogen-bond network in family A heptahelical receptors. J Mol Biol 380:648-655

33. Imai H, Kojima D, Oura T, Tachibanaki S, Terakita A, Shichida Y (1997) Single amino acid residue as a functional determinant of rod and cone visual pigments. Proc Natl Acad Sci USA 94:2322-2326

34. Bartl FJ, Vogel R (2007) Structural and functional properties of metarhodopsin III: recent spectroscopic studies on deactivation pathways of rhodopsin. Phys Chem Chem Phys 9:1648-1658

35. Vogel R, Siebert F (2002) Conformation and stability of alphahelical membrane proteins. 2. Influence of $\mathrm{pH}$ and salts on stability and unfolding of rhodopsin. Biochemistry 41:3536-3545

36. Engeszer RE, Patterson LB, Rao AA, Parichy DM (2007) Zebrafish in the wild: a review of natural history and new notes from the field. Zebrafish 4:21-40

37. Achiha A (2006) Catch depth of ocellate puffer Takifugu rubripes and water temperature in the western Enshu Nada. Aquac Sci 54:25-29 (in Japanese)

38. Lythgoe JN (1972) List of vertebrate visual pigments. In: Dartnall HJA (ed) Photochemistry of vision, handbook of sensory physiology, vol VII/I. Springer, Berlin Heidelberg New York, pp 604-624

39. Ziv L, Tovin A, Strasser D, Gothilf Y (2007) Spectral sensitivity of melatonin suppression in the zebrafish pineal gland. Exp Eye Res 84:92-99

40. Pierce LX, Noche RR, Ponomareva O, Chang C, Liang JO (2008) Novel functions for Period 3 and Exo-rhodopsin in rhythmic transcription and melatonin biosynthesis within the zebrafish pineal organ. Brain Res 1223:11-24

41. Isorna E, El M'Rabet A, Confente F, Falcón J, Muñoz-Cueto JA (2009) Cloning and expression of arylalkylamine $N$-acetyltranferase-2 during early development and metamorphosis in the sole Solea senegalensis. Gen Comp Endocrinol 161:97-102

42. Parry JW, Peirson SN, Wilkens H, Bowmaker JK (2003) Multiple photopigments from the Mexican blind cavefish, Astyanax fasciatus: a microspectrophotometric study. Vision Res 43:31-41

43. Toyama M, Hironaka M, Yamahama Y, Horiguchi H, Tsukada O, Uto N, Ueno Y, Tokunaga F, Seno K, Hariyama T (2008) Presence of rhodopsin and porphyropsin in the eyes of 164 fishes, representing marine, diadromous, coastal and freshwater species-a qualitative and comparative study. Photochem Photobiol 84:996-1002

44. Burns ME, Baylor DA (2001) Activation, deactivation, and adaptation in vertebrate photoreceptor cells. Annu Rev Neurosci 24:779-805

45. Vu TQ, McCarthy ST, Owen WG (1997) Linear transduction of natural stimuli by dark-adapted and light-adapted rods of the salamander, Ambystoma tigrinum. J Physiol 505(Pt 1):193-204

46. Sakurai K, Onishi A, Imai H, Chisaka O, Ueda Y, Usukura J, Nakatani K, Shichida Y (2007) Physiological properties of rod photoreceptor cells in green-sensitive cone pigment knock-in mice. J Gen Physiol 130:21-40 
47. Baylor DA, Nunn BJ, Schnapf JL (1984) The photocurrent, noise and spectral sensitivity of rods of the monkey Macaca fascicularis. J Physiol (Lond) 357:575-607

48. Robinson PR, Cohen GB, Zhukovsky EA, Oprian DD (1992) Constitutively active mutants of rhodopsin. Neuron 9:719-725

49. Ramon E, del Valle LJ, Garriga P (2003) Unusual thermal and conformational properties of the rhodopsin congenital night blindness mutant Thr-94-> Ile. J Biol Chem 278:6427-6432

50. Ramon E, Mao X, Ridge KD (2009) Studies on the stability of the human cone visual pigments. Photochem Photobiol 85:509-516

51. Meissl H, Ekström P (1988) Dark and light adaptation of pineal photoreceptors. Vis Res 28:49-56

52. Hartwig H-G, van Veen $T$ (1979) Spectral characteristics of visible radiation penetrating into the brain and stimulating extraretinal photoreceptors. J Comp Physiol A: Neuroethol, Sens, Neural, Behav Physiol 130:277-282

53. Nordtug T, Berg O (1990) Optical properties of the pineal window of Atlantic salmon (Salmo salar L.). Fish Physiol Biochem 8:541-546

54. Thorarensen H, Clarke WC, Farrell AP (1989) Effect of photoperiod and various intensities of night illumination on growth and seawater adaptability of juvenile coho salmon (Oncorhynchus kisutch). Aquaculture 82:39-49

55. Migaud H, Taylor JF, Taranger GL, Davie A, Cerdá-Reverter JM, Carrillo M, Hansen T, Bromage NR (2006) A comparative ex vivo and in vivo study of day and night perception in teleosts species using the melatonin rhythm. J Pineal Res 41:42-52

56. Kusmic C, Barsanti L, Passarelli V, Gualtieri P (1993) Photoreceptor morphology and visual pigment content in the pineal organ and in the retina of juvenile and adult trout, Salmo irideus. Micron 24:279-286

57. Ekström P, Meissl H (1997) The pineal organ of teleost fishes. Rev Fish Biol Fish 7:199-284

58. Tabata M, Suzuki T, Niwa H (1985) Chromophores in the extraretinal photoreceptor (pineal organ) of teleosts. Brain Res 338:173-176
59. Shichida Y, Imai H (1998) Visual pigment: G-protein-coupled receptor for light signals. Cell Mol Life Sci 54:1299-1315

60. Golobokova EY, Govardovskii VI (2006) Late stages of visual pigment photolysis in situ: cones vs. rods. Vis Res 46:2287-2297

61. Imai H, Terakita A, Tachibanaki S, Imamoto Y, Yoshizawa T, Shichida Y (1997) Photochemical and biochemical properties of chicken blue-sensitive cone visual pigment. Biochemistry 36:12773-12779

62. Nordström K, Larsson TA, Larhammar D (2004) Extensive duplications of phototransduction genes in early vertebrate evolution correlate with block (chromosome) duplications. Genomics 83:852-872

63. Shen YC, Raymond PA (2004) Zebrafish cone-rod (crx) homeobox gene promotes retinogenesis. Dev Biol 269:237-251

64. Chen C-K, Woodruff ML, Chen FS, Shim H, Cilluffo MC, Fain GL (2010) Replacing the rod with the cone transducin subunit decreases sensitivity and accelerates response decay. J Physiol 588:3231-3241

65. Kusmic C, Marchiafava PL, Strettoi E (1992) Photoresponses and light adaptation of pineal photoreceptors in the trout. Proc R Soc Lond B Biol Sci 248:149-157

66. Kuwayama S, Imai H, Hirano T, Terakita A, Shichida Y (2002) Conserved proline residue at position 189 in cone visual pigments as a determinant of molecular properties different from rhodopsins. Biochemistry 41:15245-15252

67. Goncalves JA, South K, Ahuja S, Zaitseva E, Opefi CA, Eilers M, Vogel R, Reeves PJ, Smith SO (2010) Highly conserved tyrosine stabilizes the active state of rhodopsin. Proc Natl Acad Sci USA 107:19861-19866

68. Lamb TD, Collin SP, Pugh EN (2007) Evolution of the vertebrate eye: opsins, photoreceptors, retina and eye cup. Nat Rev Neurosci 8:960-976

69. Davies WL, Collin SP, Hunt DM (2009) Adaptive gene loss reflects differences in the visual ecology of basal vertebrates. Mol Biol Evol 26:1803-1809 